\title{
Network Distance and Fatal Outcomes among Gunshot Wound Victims
}

\author{
Giovanni M. Circo Ph.D., University of New Haven
}

Andrew P. Wheeler Ph.D., University of Texas at Dallas

\begin{abstract}
Despite nation-wide decreases in crime, urban gun violence remains a serious and pressing issue in many cities. Victim survival in these incidents is often contingent on the speed and quality of care provided. Increasingly, new research has identified the role that specialized trauma care plays in victim survival from firearm-related injuries. Using nearly four years of data on shooting victimizations in Philadelphia we test whether distance to the nearest level 1 trauma center is associated with victim survival. We employ different distance measures based on street network distances, drive-time estimates, and Euclidian distance - comparing the predictive accuracy of each. Our results find that victims who are shot farther from trauma centers have an increased likelihood of death, and drive time distances provide the most accurate predictions. We discuss the practical implications of this research as it applies to urban public health.
\end{abstract}

Keywords: gun-violence, street-network, emergency-response, fatality

Materials to replicate the results can be found at https://www.dropbox.com/sh/jzxeays1aro9690/AAAVu1AGIyPohtwcdTx9vR Ja?dl=0. 


\section{Introduction}

The costs of gun violence are well documented. In 2016 alone, over 38,000 Americans died of gun-related injuries (Vlahov, 2018). Setting aside suicides, 15,549 individuals were killed by firearms in 2017. While firearm-related deaths represent a nation-wide problem, urban gun violence reflects the convergence of poverty, racial segregation, and weapon availability (Friedson \& Sharkey, 2015; Roberto, Braga, \& Papachristos, 2018). Urban areas disproportionately contribute to the overall number of firearm deaths and subsequently are the focus of many gun violence prevention initiatives (Braga, Papachristos, \& Hureau, 2010). While law enforcement is concerned with preventing and apprehending individuals involved in gun violence, the treatment of victims is of equal importance. Indeed, access to medical care is critical among those who are injured in violent incidents - with the first 24 hours often seen as especially important (Kellerman, 2004). Improved medical care has meant that victim survival from even severe firearm injuries is no longer automatically fatal (Joseph, et al., 2014; Manley, et al., 2018). Consequently, access to medical care - such as specialized trauma care - is an important factor in victim survival. Our paper focuses on this particular question: that is, the role that level 1 trauma centers play in the "chain of survival" for victims with gunshot wounds (Nicholl, 1999). To answer this question, we analyze shooting victimization data from Philadelphia, PA between 2015 and 2019 on 5,580 individuals. Our analysis addresses on two primary questions:

1. Is victim survival associated with distance to the nearest level 1 trauma center?

2. Does the estimate of survival depend on the type of distance measure?

While the first question has been addressed both directly and indirectly in several studies (e.g.: Crandall, et al., 2013; Seamon, et al., 2017; Beard, et al., 2019; Circo, 2019), the second 
question focuses on a more specific concern. Most prior studies have relied on straight-line, "as the crow flies" distance from the site of the victimization to the nearest trauma center. This measure potentially ignores several important factors related to transit time, including the distribution of the road network, road speeds, and time-varying traffic levels (Caniglia et al., 2019). To test this, we utilize data from the Google distance matrix API which allows the estimation of real-world drive times over road networks. We contrast our model estimates using network drive time, network distance, and Euclidean distance as it pertains to predictive accuracy and model coefficients. We find distance to the nearest level 1 trauma center is inversely related to victim survival, with victims who are shot farther from trauma centers having a higher probability of death. When comparing models, we find that network drive time provides more accurate predictions compared to distance-only measures. In our results section we discuss the implications of this study as it pertains to urban gun violence and the optimal distribution of critical health care.

\section{Urban Gun Violence, Trauma Centers, and Victim Survival}

Urban gun violence represents both a public safety and public health problem costing cites and municipalities hundreds of millions of dollars each year (Cook \& Ludwig, 2000). The cost of treating a single gunshot wound victim can be upwards of $\$ 45,000$, not including the costs post-treatment (Bartlett, 2003). Beyond the simple monetary cost of gun violence is the substantial personal cost of physical injury and death of the victim, along with the negative impact to communities - the cost of which is difficult to quantify. While the primary goal of police and public health officials should be the prevention of gun violence before it happens, a secondary goal should be the minimization of harm after it occurs (Nicholl, 1999; Kellerman, 2004). Crucial among these is access to timely medical care immediately after being shot 
(Kellerman, 2004). In urban areas, hospitals with specialized medical staff and facilities play an important role in victim survival. Level 1 trauma centers represent the highest level of care available for victims with traumatic injuries. These locations must meet a number of requirements including 24 -hour coverage by surgeons and specialists, providing prevention to local communities, and treating a minimum number of severely injured patients each year (American Trauma Society, 2019). In cases of critical injuries, such as gunshot wounds, level 1 trauma centers are the preferred patient destination (Lale, Krajewski, \& Friedman, 2017). A nationwide study found that patients treated at hospitals with level 1 trauma centers had lower inhospital mortality rates than similar patients at non-trauma center hospitals (MacKenzie, et al., 2006). Therefore, access to these facilities among gunshot wound victims is especially important. With this question in mind, a wide variety of studies have examined whether victim survival in gunshot wound cases is contingent on distance to the nearest trauma center.

In urban areas gun violence is generally stably concentrated in a small number of neighborhoods (Braga, Papachristos, \& Hureau, 2010). These areas disproportionately contribute to overall levels of violence, and generate a disproportionate number of victims as well (Braga, et al., 2008). The question, therefore, is whether the distribution of hospitals with level 1 trauma facilities impacts victim survival - taking into consideration the spatial dynamics of urban areas. If victim survival is, indeed, contingent on the time to the nearest trauma center, then disparities in outcomes might be spatially concentrated (Wandling, Behrens, Hsia, \& Crandall, 2016). In Chicago researchers found evidence of so-called "trauma deserts", which were areas more than five miles away from the nearest trauma center (Crandall, et al., 2013). Victims in these areas had an increased risk of death by about $23 \%$ compares to those who were not more than five miles away. Many of these underserved areas were distributed on Chicago's South-East side, 
suggesting a need for increased access (Wandling, et al., 2016). A further follow-up study in Chicago found that nearly $30 \%$ of gunshot wound victims were treated in a non-designated trauma center - what the authors classified as "under-triage" (Lale, Krajewski, \& Friedman, 2017). This research suggested that lack of access to appropriate care could lead to worse outcomes among victims.

There has been a limited number of other analyses examining the effect of distance to trauma centers on gunshot wound mortality. Among two studies in Detroit, one found that much of the variation in trauma patient outcomes was related to the hospital at which the victim was treated, and not neighborhood-level risk factors (Sall, Hayward, Fessler, \& Edhayan, 2018). The authors highlighted the geographical differences in treatment outcomes based on access to hospital care. Another recent study by Circo (2019) examined the distribution of gunshot wound mortality among neighborhoods in Detroit. This study found that clusters of census blocks nearest to trauma centers had significantly lower rates of deaths from gunshot wounds. However, the data in this study was not sufficient to disaggregate to the individual-level. A Philadelphiabased study examining firearm injury intake at trauma centers found that clustered arrivals (gunshot wound victims arriving within 15 minutes of each other) were relatively common (Beard, et al., 2019). While mass shootings gain significant notoriety in the media, this study highlighted the victim burden which trauma centers routinely face. In general, this small collection of studies highlight two concerns: the volume of gun violence in some urban areas, and the challenges of providing adequate treatment.

At the incident level, several studies have elaborated covariates regarding victim survival in shootings. Variables such as age and incident location are important - with older victims and victims shot indoors more likely to die of their injuries, or suffer more serious injuries 
(Grommon \& Rydberg, 2014; Braga \& Cook, 2018). For obvious reasons, victims who are struck in less critical body areas (such as the arms, legs, or other extremities) are less likely to die of their injuries than those who are struck in the torso or head. While trauma care for gunshot wound injuries has improved, victims who are struck in the head die upwards of $50-80 \%$ of the time (Joseph, et al., 2014). Because shootings that take place outdoors often involve victims and offenders who are farther apart, the probability of a fatal wound is lessened somewhat (Braga \& Cook, 2018). Therefore, the characteristics of the incident (such as a drive-by shooting versus a domestic violence shooting) are likely to affect the victim's outcome. These differences may greatly sway the probability of survival, so adjusting for these covariates is important when isolating the impact of other effects - such as hospital transport time. A number of municipalities (including Philadelphia) maintain open-source databases containing individual-level data on shooting victims, including the circumstances of the incident, and the victims' characteristics. Applying this type of data stands to improve what is known about gun violence and factors which lead to victim survival or death -both at the incident and neighborhood-level.

\section{Data and Methods}

The primary data for analysis are open-data reports of shooting victims in Philadelphia from $1 / 1 / 2015$ through $6 / 20 / 2019^{1}$. From this dataset we obtain whether the shooting victim ultimately died based on the incident report, as well as the geographic location of the incident (aggregated to the center of the street segment to provide some anonymity). Given the open data sources, we have provided open source code to replicate the findings.

\footnotetext{
${ }^{1}$ Data is publicly available at https://www.opendataphilly.org/dataset/shooting-victims
} 
The main independent variables of interest are estimates of distances to the nearest level 1 trauma center. These are calculated, and models are compared for three different measures: Euclidean 'as the crow flies' distance between shootings and hospitals, street road network distance, and average estimated drive times over the street network. Euclidean distances based on the spherical coordinates of the shooting location and the trauma center are calculated using the Vincenty formula. The street network distances and drive times are calculated via the Google distance matrix $\mathrm{API}^{2}$. In particular, the average estimated drive times are times based on long term averages of actual drive times over those road network segments. We suspect that drive times based on the road network are more directly pertinent to estimating the probability of death, relative to simple Euclidean metrics (Crandall et al., 2013, Circo, 2019). We calculate all three metrics in reference from each shooting to each hospital, and then model the closest of the three for each measure. In many cases hospitals have closer drive times but further away Euclidean or street network distances.

Although it would be preferable to directly have ambulance response times (e.g. Crandall et al., 2013; O'Keefe et al., 2010), we suspect that the drive times should provide similar marginal effect estimates (e.g. the effect of being 1 minute further away on the road network should closely translate to 1 minute longer total response-to-hospital times for ambulances). Specifically, while these metrics do not take into account the time it takes to initially respond to an incident, they should be highly correlated. Thus these estimates are likely more directly pertinent to estimating the effect of improving ambulance response times to shooting incidents than Euclidean distance metrics.

\footnotetext{
${ }^{2}$ https://developers.google.com/maps/documentation/distance-matrix/start
} 
There are a total of five non-pediatric level 1 trauma centers within Philadelphia. While victims may be sent to pediatric trauma centers or to other hospitals without trauma centers, it appears to be a much lower frequency than the non-pediatric centers in Philadelphia (Beard et al., 2019). Figure 1 displays the locations of those trauma centers, along with a map illustrating the spatial distribution of shootings with a victim. Temple hospital is closest to the densest area of shootings, with a hexagon grid of over 200 victimizations over the three and a half year period - around 3 kilometers away.

\section{[INSERT FIGURE 1 HERE]}

Another important factor included in the model are the locations of where an individual was shot on their body. Wound locations are recorded as a plain text field in the Philadelphia shooting victim dataset, and we recoded those fields into the final wound locations of head, torso, extremities, or multiple wound locations (Grommon \& Rydberg, 2015). The other independent variables of interest that are available in the model include the age of the victim, the sex of the victim, the race of the victim, and whether the shooting occurred outdoors. The age of the victim has obvious implications for the probability of survival, but the other demographic factors may also be associated with the nature of the shooting incident (Hipple \& Magee, 2017). Subsequently they are potentially important to control for to prevent confounds when examining other factors (Wiebe, 2003). Finally, we calculated the number of clustered arrivals as the number of shootings within the prior 15 minutes and being closest to the same hospital based on drive time distances (Beard et al., 2019).

Table 1 displays descriptive statistics for the variables used in the analysis. The original shooting dataset contained 5,740 cases, of which we eliminated 160 for analysis (less than 3\% of the total sample). These were mostly due to missing spatial locations where the shootings took 
place $(n=73)$, as well as cases that were missing data on wound location $(n=56)$, age $(n=30)$, sex $(n=4)$, or race $(n=4)$. Our final dataset contained 5,580 cases.

\section{[INSERT TABLE 1 HERE]}

The model we estimate is a logistic regression model, predicting the probability of a death subsequent to the shooting based on the distance to the nearest level 1 trauma center, along with the other independent variables previously listed. Our equation predicting the probability of death, $p$, can be written as:

$$
\begin{aligned}
\operatorname{logit}(p)=\beta_{0} & \left.+\beta_{1}(\text { Distance to Nearest Trauma })+\beta_{2} \text { (Injury Torso }\right) \\
& \left.+\beta_{3}(\text { Injury Extremities })+\beta_{4}(\text { Injury Multiple })+\beta_{5} \text { (Outdoors }\right) \\
& \left.\left.+\beta_{6}(\text { Clustered Arrivals })+\beta_{7}(\text { White })+\beta_{8} \text { (Male }\right)+\beta_{9} \text { (Age }\right)
\end{aligned}
$$

$\beta_{1}$ is then the main effect of interest, and we estimate this same model using three different distance metrics (drive time, street network distance, and Euclidean distance). We subsequently evaluate the fit of those three different measures using the AIC and BIC statistics, as well as leave-one-out cross validation error estimates (Harrell, 2001).

Additional control variables are coefficients $\beta_{2}$ through $\beta_{9}$, adjusting for wound location, shooting location, clustered hospital arrivals, and victims' race, sex, and age. For the categorical variable of wound location, we use wounds to the head as the referent category, with dummy variables representing wounds to the torso, extremities, or multiple locations. We also include dummy variables for being a male, being white, and a linear effect for age. The continuous variables of age and distance are mean centered before being estimated, which only impacts the interpretation of the intercept term, $\beta_{0}$.

\section{Results}


Figure 2 displays the marginal effects of distance to the nearest level 1 trauma center (in terms of estimated travel time on the road network), broken down by individual wound locations. The blue line shows the fit of the simple logistic model, and the red line (and pink 95\% confidence interval error bars around the mean), show the non-linear fit based on a generalized additive model. The grey circles show the actual data, binned to every 2.5 minutes and plotted at the mean number of fatalities, with bigger circles illustrating a larger number of cases. The motivation for this plot was to determine if travel time had a non-linear effect on the probability of death (Harrell, 2001), as well as potential interactions depending on the severity of the wound. For example, the probability of death may increase up to 5 minutes away, and then flatten off. Such a pattern could occur if cases had a polarized distribution, with a proportion of cases where time does not matter at all, and others where only a very short response would have prevented a fatality.

\section{[INSERT FIGURE 2 HERE]}

While the non-linear red line fluctuates in several of the sub-panels, the error bars around the non-linear effect always contain the linear fit estimate. Based on this exploratory data analysis, there is little evidence of non-linear effects based on the travel time to the nearest level 1 trauma center (plots based on the other distances yielded similar conclusions). Therefore, subsequent logistic regression models predicting the probability of death include only linear terms.

Table 2 displays the results of the logistic regression equations, showing models that swap the ultimate distance term of interest. Model 1 uses the drive time estimates, Model 2 uses street network distances, and Model 3 uses Euclidean 'as the crow flies' distance. All three models produce a similar interpretation, with those shootings further away resulting in an 
increased probability of death. Additionally the models show that having a wound location in the torso, extremities, or multiple locations have a much lower probability of death, relative to the referent category of being shot in the head. Additionally being older results in a higher probability of death, and being shot outside results in a lower probability of death. Although these effects are smaller in magnitude relative to the wound location. The effect of clustered events has a negative coefficient, replicating the finding of decreased mortality in Beard et al. (2019), but is not significant $(\mathrm{p}=0.114)$. Being male also results in a marginally significant increase in mortality $(\mathrm{p}=0.049)$. Race does not have a significant effect on the probability of death $(\mathrm{p}=0.25)$

\section{[INSERT TABLE 2 HERE]}

Based on the large direct effects of wound location, we also examined the interactions between the wound location and the drive time distance. Those interaction effects were not individually statistically significant, and they produced overall a worse fit compared to the model without interactions. As such we only report the models without those interaction effects. For the drive time distance model, we also examined the spatial autocorrelation of the deviance residuals. Using a row normalized inverse distance weighted matrix, with events at the same location calculated as a distance of 1, we calculated global Moran's I (Anselin, 1995). The overall estimate of the spatial auto-correlation was less than 0.01 , with a p-value of 0.022 based on 999 permutations (Anselin, 1995).2. While the p-value indicated a statistically significant difference from 0 , the very low level of auto-correlation suggests it is unlikely to greatly impact the results. Subsequently there is no reason to believe the model results are biased due to other unaccounted for spatial factors. 
A key question of our study was the comparison of three different distance measures. As stated before, most prior research relies on straight-line Euclidean distance from the incident location to the hospital. Our aim was to determine whether estimating distances along the street network (network distance) or using historical estimates of drive time (network time) was superior to straight line distances. Conceptually, network distance or network time is a more internally valid measure because it reflects real-world limitations of travel (i.e. based on roads, natural barriers, etc.). We used three estimates of approximate out-of-sample performance: Akaike information criterion (AIC), Bayesian information criterion (BIC), and leave-one-out cross validated error (CVE). AIC is an information criterion which provides an estimate of outof-sample deviance, while BIC attempts to identify the "true" model using the model's average likelihood (Burnham \& Anderson, 2004). In practice, both AIC and BIC tend to come to similar results when the sample size $n$ is much larger than the number of parameters $k$. Leave-one-out CVE is a common method used to estimate out-of-sample performance by iteratively dropping one observation, then using the remaining data to make a prediction for that single point. This process is repeated $n$ times yielding an estimate of out-of-sample predictive error for the model (Harrell, 2001).

Using these model comparison criterion we identified that Model 1, using network time, provided a marginally better fit than both network distance or Euclidean distance for all three model fit statistics $(\mathrm{AIC}=4071.4, \mathrm{BIC}=4137.6, \mathrm{CVE}=0.11979)$. This provides evidence that modeling the spatial dynamics of urban street networks is an improvement over more simple straight-line distances. The estimated effect of one minute of travel along the street network was an increased likelihood of death by approximately $2 \%(\beta=0.022, p=0.26)$. In contrast, the estimated effect for one kilometer of travel along the street network was $3 \%(\beta=0.030, p=$ 
$0.067)$ and $4 \%$ for Euclidean distance $(\beta=0.042, p=0.046)$. Based on model fit statistics we focus the remainder of our results derived from Model 1.

Figure 3 displays the marginal effects of being further away in drive time distance, broken down by each wound location type. The bands around each prediction plot the pairwise $95 \%$ confidence interval of those mean predictions. These predictions are also generated by setting the sex to male, age to 28.7, the location to outside, and the race to Black (either the modal categories or the means). Being shot in the extremities always produces a low probability of death, while being shot in the head is the highest. In cases of more serious wounds, the marginal effects of being near a level 1 trauma center matter more. For example, the probability of death when being shot in the head is just 0.49 when the travel time distance is 10 minutes, whereas it is 0.54 when the travel time is 20 minutes. Constructing the same contrast for wounds in the torso, one goes from a probability of 0.22 to 0.26 . Wounds to multiple locations result in very similar predicted probabilities of deaths to those in the torso.

\section{[INSERT FIGURE 3 AND FIGURE 4 HERE]}

Figure 4 displays a map showing the predicted probability changes based on estimated travel time distances on a regular grid over the city for wounds to the torso. (Other variables are held at the same constants as those in Figure 3) The brighter areas indicate bands very nearby the hospital in terms of drive times. Cross referencing this with the prior map showing the distribution of shootings, it appears those being shot in the west and southwest Philadelphia neighborhoods are not as well situated in terms of drive times to level 1 centers, compared to the hot spot of shootings in the more eastern/central areas of the city (closer to the Kensington neighborhood). Depending on the where you are located when you are shot, it can sway the predicted probability of death by 14 percentage points in this example. For instance, in some of 
the northern parts of Philadelphia the predicted probability of a fatality is over $32 \%$, whereas the same incident nearby a hospital in central Philadelphia only has an $18 \%$ probability of fatality.

\section{Discussion}

Based on prior research we expected to find some evidence linking the distance to a trauma center with victim survival (Crandall, et al., 2013; Sall et al., 2018; Circo 2019). Indeed, a wealth of studies have illustrated the importance that specialized trauma care plays for critically injured individuals (Lale, Krajewski, \& Friedman, 2017). With improvements in medicine, surgery, and trauma care, gunshot wound victims have increasingly better outcomes (Manley, et al., 2018). However, a key link in this chain is improving victim access to care. Our study and others find that geographic disparities in access to trauma centers is likely to cause an excess number of deaths among victims who might have otherwise survived. Because addressing gun violence is a multi-faceted issue, treating victims remains one of many important issues.

The results of our findings can be used in two particular ways. One is that the results can be used to inform strategies that attempt to reduce the drive time of ambulances (Chanta et al., 2011). The estimates we provide can help siting decisions for ambulances in conjunction with real-time predictions based on where shootings are likely to occur (Hosler et al., 2018). City planners may consider the cost-benefit ratio of opening an additional hospital, given the number of lives which might plausibly be saved. Aside from creating new hospitals, focusing additional resources on areas where victims are more likely to die of injuries may be an effective intermediate measure. For instance, training officers to transport victims to the hospital when emergency medical services are not available may improve victim outcomes. In fact, this "scoopand-run" strategy has already been evaluated in Philadelphia before (Seamon, et al., 2007). 
Our results can also be used in model parameters if one is aiming to improve survivability among gunshot wound victims (Chanta et al., 2014). A noted limitation is that our model estimates can only indirectly inform such an intervention, as we do not directly calculate ambulance response times, but only estimated response times based on average street network times. Our estimates also assume rational behavior - namely, that injured victims are sent to the nearest level 1 trauma center. Limitations of this data preclude estimates of travel based on transport type (i.e. ambulance vs personal vehicle). Despite this, our study represents a unique analysis of the interaction between incident-level variables and the distribution of trauma services in an urban area.

One strength of our estimates is that they can also provide counterfactual evidence for siting decisions for trauma centers themselves, such as the likely effects for either adding or subtracting a level 1 trauma center on subsequent survivability (Crandall et al., 2016). When conducting either type of siting decision, either allocating ambulances or opening or closing a level 1 trauma center, to estimate the subsequent effects one needs to have model-based estimates of how increased/decreased travel times will impact the subsequent survivability of gunshot patients. Using actual drive time estimates we believe is likely to provide more realistic estimates than those based simply on Euclidean distances. But when extrapolating in what-if type scenarios, network based measures of drive time estimates likely provide a reasonable estimate of survivability in making future decisions about the spatial provision of emergency services. 


\section{References}

American Trauma Society. (2019). Trauma Center Levels Explained. Retrieved from: https://www.amtrauma.org/page/traumalevels?

Anselin, L. (1995). Local indicators of spatial association-LISA. Geographical analysis, 27(2), 93-115.

Bartlett, C. S. (2003). Clinical update: gunshot wound ballistics. Clinical Orthopaedics and Related Research ${ }^{\circledR}, 408,28-57$.

Beard, J. H., Resnick, S., Maher, Z., Seamon, M. J., Morrison, C. N., Sims, C. A., ... \& Goldberg, A. J. (2019). Clustered arrivals of firearm-injured patients in an urban trauma system: a silent epidemic. Journal of the American College of Surgeons.

Braga, A. A., Papachristos, A. V., \& Hureau, D. M. (2010). The concentration and stability of gun violence at micro places in Boston, 1980-2008. Journal of Quantitative Criminology, 26(1), 33-53.

Caniglia, E. C., Zash, R., Swanson, S. A., Wirth, K. E., Diseko, M., Mayondi, G., ... \& KponeeShovein, K. Z. (2019). Methodological Challenges When Studying Distance to Care as an Exposure in Health Research. American Journal of Epidemiology.

Chanta S., Mayorga, M.E., \& McLay, L.A. (2014). The minimum p-envy location problem with requirement on minimum survival rate. Computers \& Industrial Engineering, 74, 228239. 
Chanta, S., Mayorga, M.E., Kurz M.E, \& McLay, L.A. (2011). The minimum p-envy location problem: A new model for equitable distribution of emergency resources. IIE Transactions on Healthcare Systems Engineering, 1(2), 101-115.

Circo, G. M. (2019). Distance to trauma centres among gunshot wound victims: identifying trauma 'deserts' and 'oases' in Detroit. Injury prevention, injuryprev-2019.

Cook, P. J., \& Ludwig, J. (2000). Gun violence: The real costs. Oxford University Press on Demand.

Crandall, M., Sharp, D., Unger, E., Straus, D., Brasel, K., Hsia, R., \& Esposito, T. (2013). Trauma deserts: distance from a trauma center, transport times, and mortality from gunshot wounds in Chicago. American Journal of Public Health, 103(6), 1103-1109.

Crandall, M., Sharp, D., Wei, X., Nathens, A., \& Hsia, R.Y. (2016). Effects of closure of an urban level 1 trauma centre on adjacent hospitals and local injury mortality: A retrospective, observational study. BMJ Open, 6(5), e011700.

Friedson, M., \& Sharkey, P. (2015). Violence and neighborhood disadvantage after the crime decline. The Annals of the American Academy of Political and Social Science, 660(1), 341-358.

Grommon, E., \& Rydberg, J. (2015). Elaborating the correlates of firearm injury severity: Combining criminological and public health concerns. Victims \& Offenders, 10(3), 318340.

Harrell Jr., F. (2001). Regression modelling strategies: With applications to linear models, logistic regression, and survival analysis. Springer-Verlag: New York, NY. 
Hipple, N., \& Magee, L. (2017). The difference between living and dying: victim characteristics and motive among nonfatal shootings and gun homicides. Violence and Victims, 32(6), 977-997.

Hosler, R., Liu, X., Carter, J., \& Saper, M. (2019). RaspBary: Hawkes Point Process Wasserstein Barycenters as a Service. Available at https://www.researchgate.net/profile/George_Mohler/publication/327212089_Raspbary Hawkes_Point_Process_Wasserstein_Barycenters_as_a_Service/links/5b805ab192851c1 e122f57a4/Raspbary-Hawkes-Point-Process-Wasserstein-Barycenters-as-a-Service.pdf.

Joseph, B., Aziz, H., Pandit, V., Kulvatunyou, N., O'Keeffe, T., Wynne, J., ... \& Rhee, P. (2014). Improving survival rates after civilian gunshot wounds to the brain. Journal of the American College of Surgeons, 218(1), 58-65.

Kellermann, A. L. (2004). Treating gun violence before the 911 call. Annals of Emergency Medicine, 43(6), 743-745.

Lale, A., Krajewski, A., \& Friedman, L. S. (2017). Undertriage of firearm-related injuries in a major metropolitan area. JAMA surgery, 152(5), 467-474.

MacKenzie, E. J., Rivara, F. P., Jurkovich, G. J., Nathens, A. B., Frey, K. P., Egleston, B. L., ... \& Scharfstein, D. O. (2006). A national evaluation of the effect of trauma-center care on mortality. New England Journal of Medicine, 354(4), 366-378.

Manley, N. R., Fabian, T. C., Sharpe, J. P., Magnotti, L. J., \& Croce, M. A. (2018). Good news, bad news: An analysis of 11,294 gunshot wounds (GSWs) over two decades in a single center. Journal of trauma and acute care surgery, 84(1), 58-65. 
Nicholl, J. P. (1999). Optimal use of resources for the treatment and prevention of injuries. British Medical Bulletin, 55(4), 713-725.

Roberto, E., Braga, A. A., \& Papachristos, A. V. (2018). Closer to guns: the role of street gangs in facilitating access to illegal firearms. Journal of Urban Health, 95(3), 372-382.

Sall, L., Hayward, R. D., Fessler, M. M., \& Edhayan, E. (2018). Between-hospital and betweenneighbourhood variance in trauma outcomes: cross-sectional observational evidence from the Detroit metropolitan area. BMJ open, 8(11), e022090.

Seamon, M. J., Fisher, C. A., Gaughan, J., Lloyd, M., Bradley, K. M., Santora, T. A., ... \& Goldberg, A. J. (2007). Prehospital procedures before emergency department thoracotomy:"scoop and run" saves lives. Journal of Trauma and Acute Care Surgery, 63(1), 113-120.

Wandling, M., Behrens, J., Hsia, R., \& Crandall, M. (2016). Geographic disparities in access to urban trauma care: defining the problem and identifying a solution for gunshot wound victims in Chicago. The American Journal of Surgery, 212(4), 587-591.

Vlahov, D. (2018). Building the evidence base to prevent firearm deaths and injuries. Journal of Urban Health, 95(3), 293-294. 


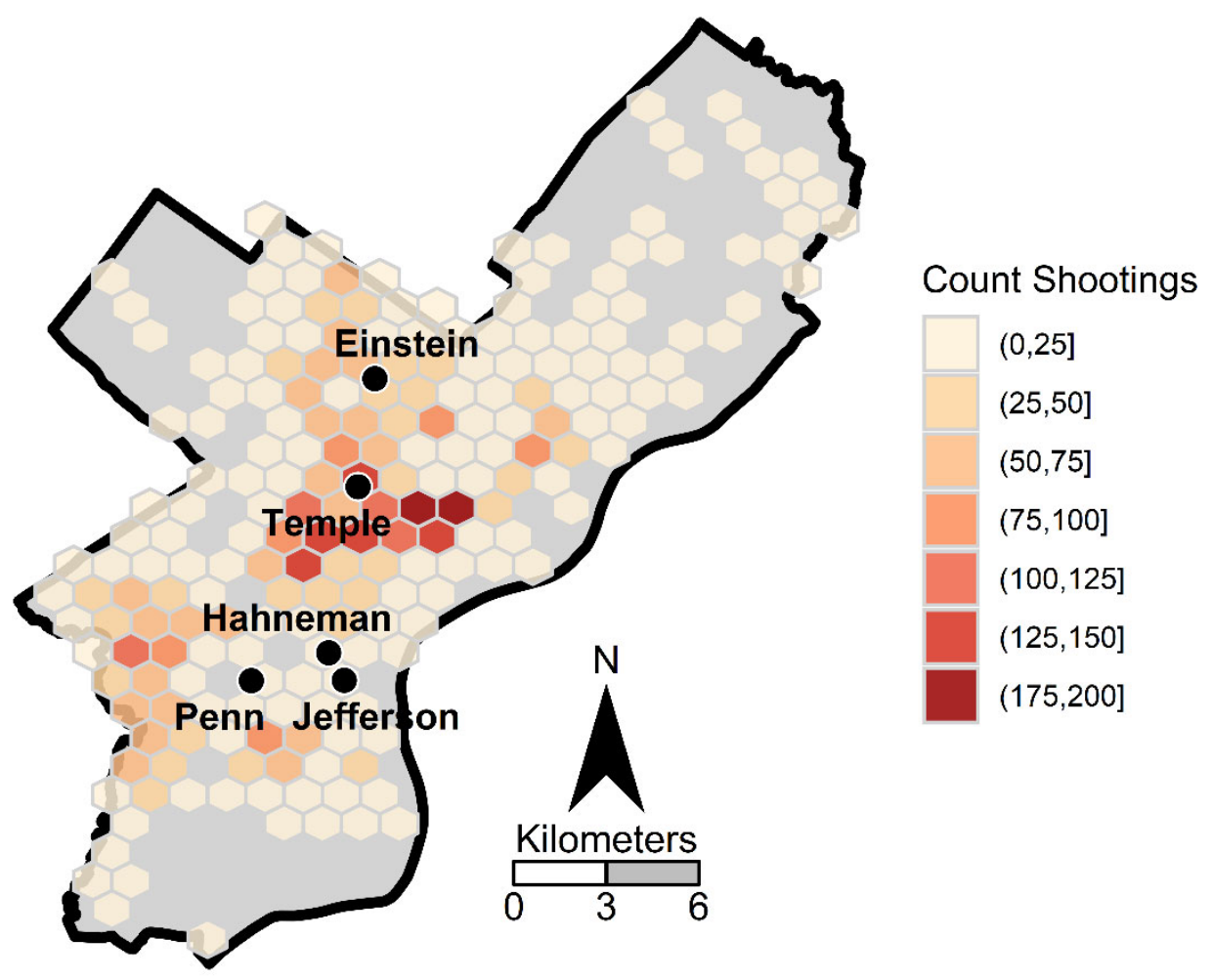

Figure 1: Count of shooting victims (from 1/1/2016 through 6/20/2019) and locations of level 1 trauma centers in Philadelphia. Hexagons have an area of 1 square kilometer. 

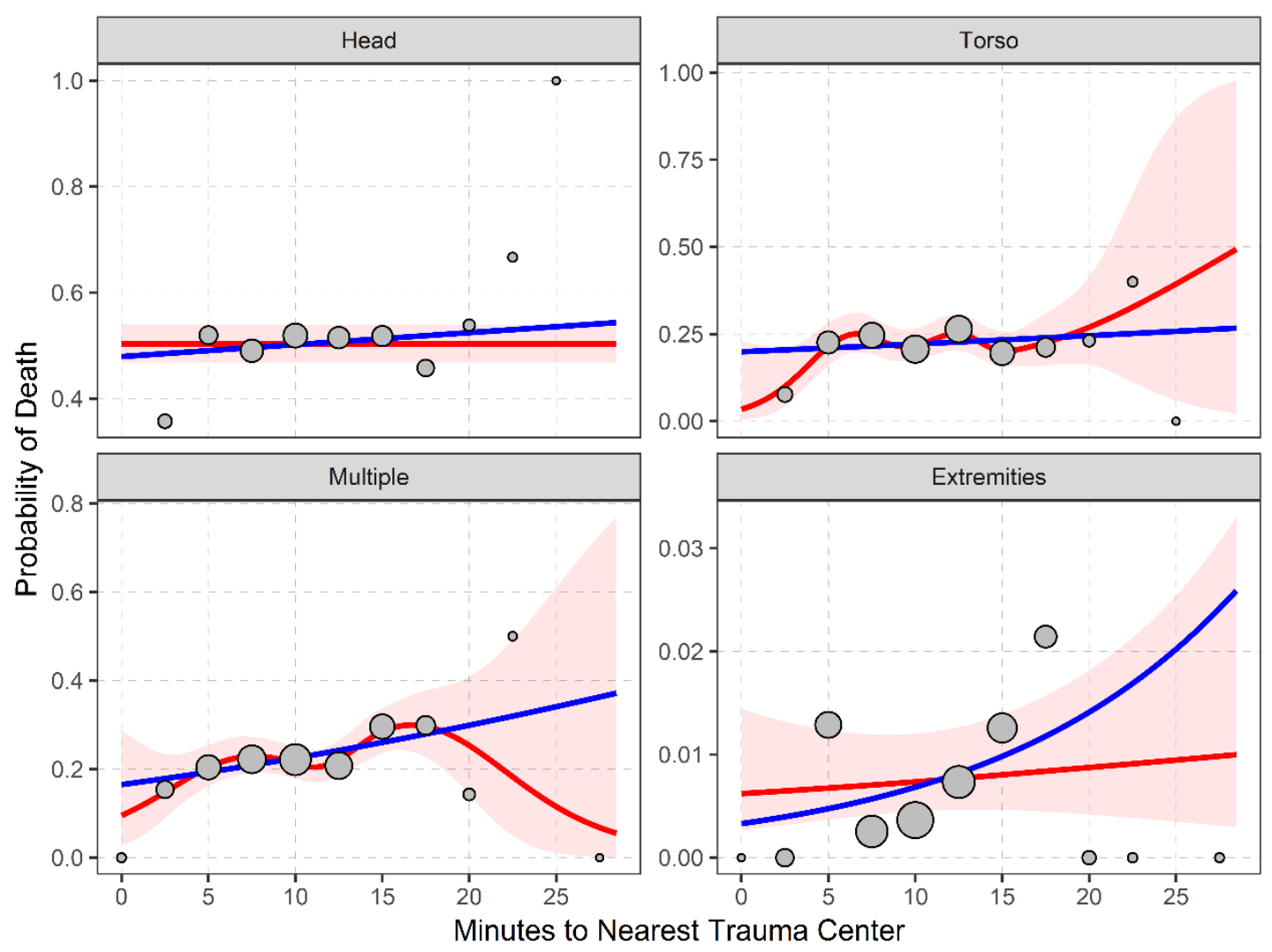

Cases

○ 100

○ 200

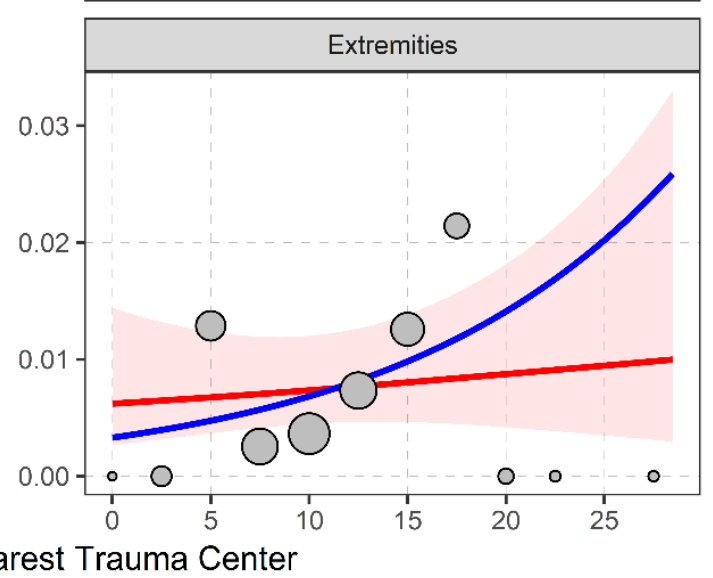

300

400

500

Figure 2: Marginal effects on number of minutes travel vs the probability of death, conditional on wound location. Straight line shows the fit for a logistic regression based on travel minutes to nearest trauma center. Curved line and error bars show fit for generalized additive model estimated non-linear effect of minutes to nearest center. Grey circles show aggregated data to every 2.5 minutes and plotted at the mean number of fatalities within that bin. 


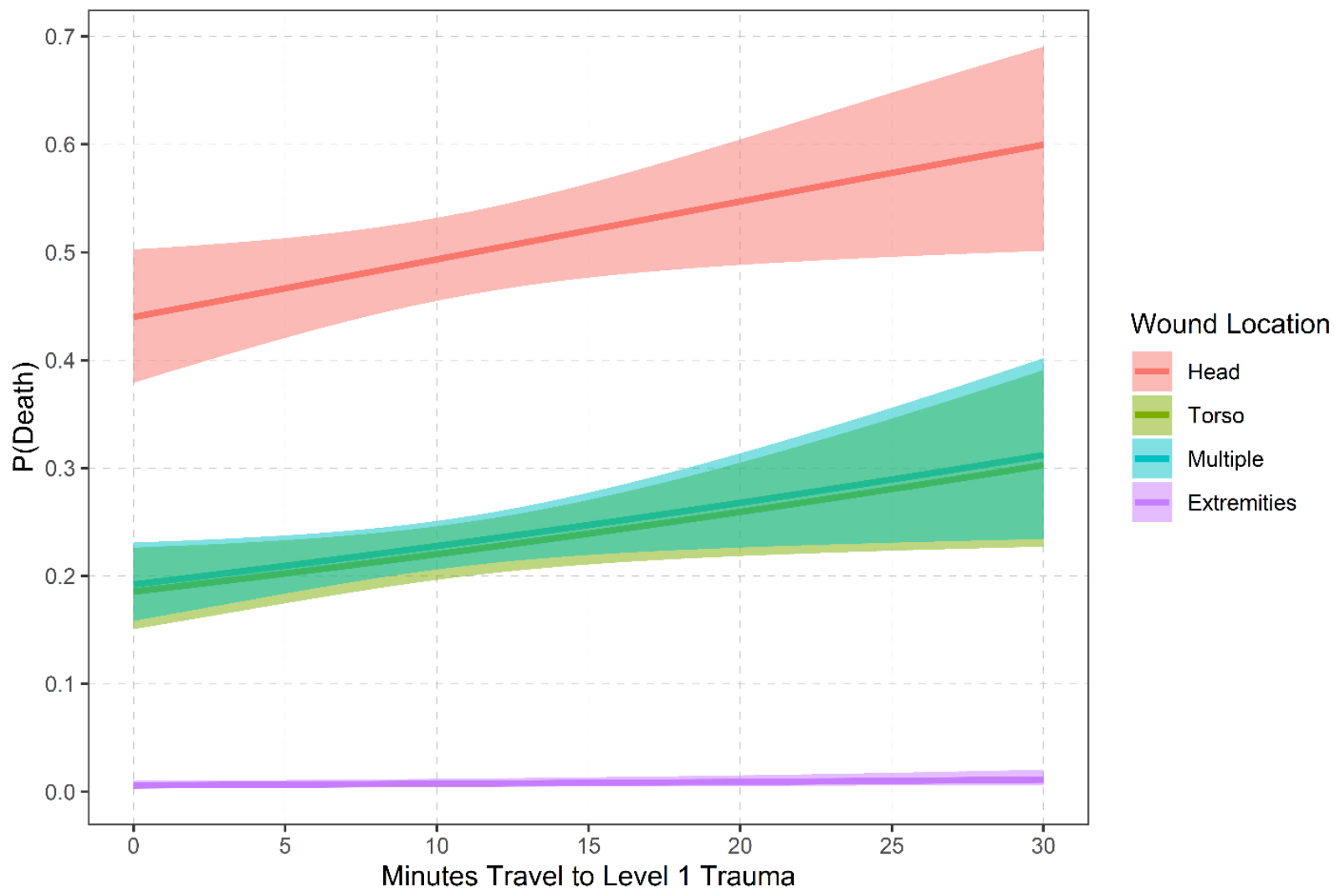

Figure 3: Predicted probability of death from gun shot given network time distances, conditional on wound location. 


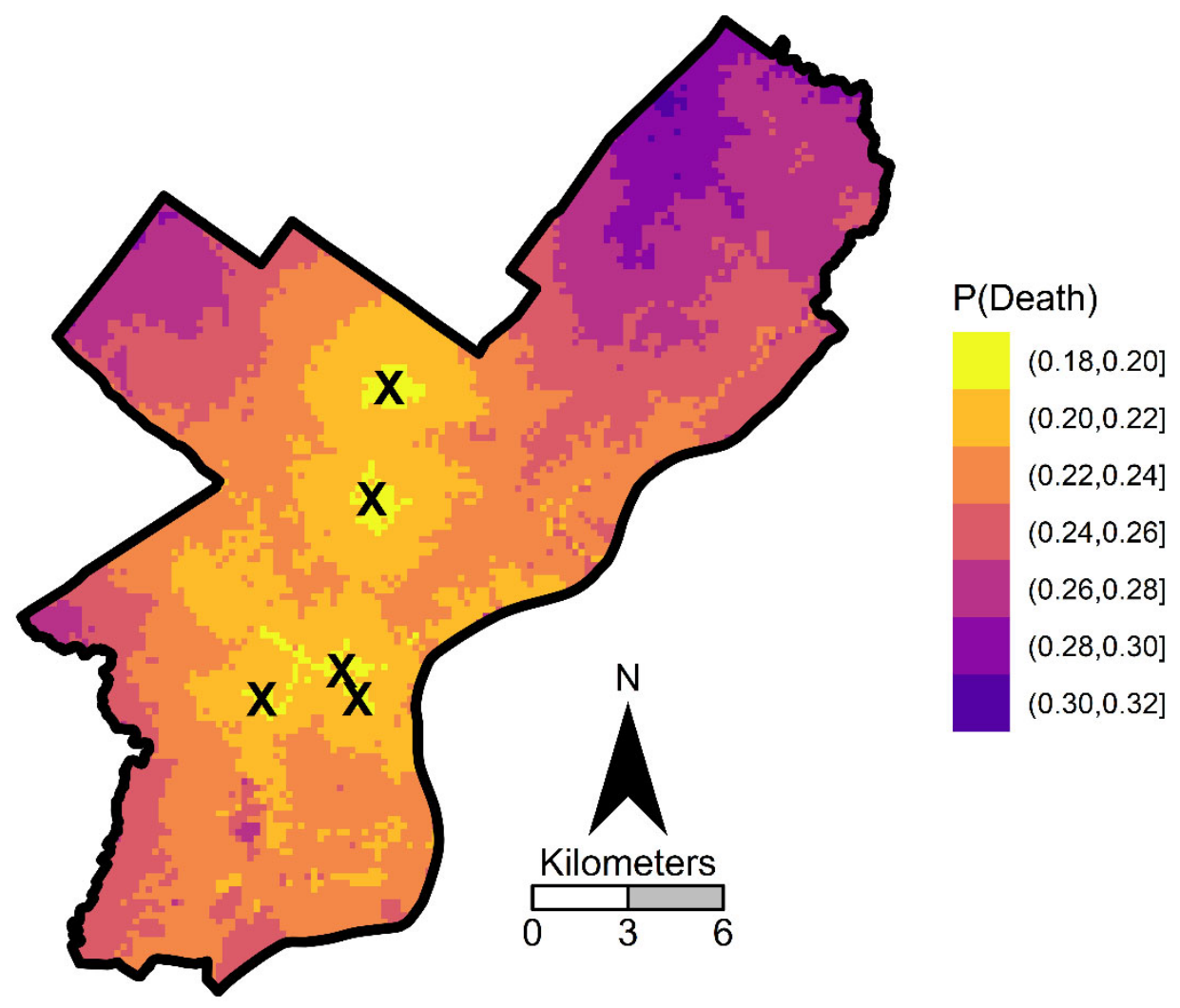

Figure 4: Isochrone map surface for the predicted probability of death based on estimated drive time distances and gun shot wound to the torso. X's show the location of level 1 trauma centers. 


\section{Table 1. Descriptive Statistics}

\begin{tabular}{lr} 
Variable & Mean/Prop. \\
\hline Distance Measure & \\
\hline Network Time $(\mathrm{min})$ & 10.50 \\
Network Distance $(\mathrm{km})$ & 3.84 \\
$\quad$ Euclidean Distance $(\mathrm{km})$ & 2.93 \\
Injury Location & \\
\hline Head & 0.14 \\
Torso & 0.22 \\
Multiple & 0.26 \\
Extremities & 0.38 \\
Victim \& Situational & \\
Incident Outdoors & 0.94 \\
Clustered Arrivals $(15 \mathrm{~min})$ & 0.02 \\
Race White & 0.17 \\
Race Black & 0.83 \\
Sex Male & 0.92 \\
Sex Female & 0.08 \\
Age & 28.70 \\
\hline $\mathrm{N}=5580$ &
\end{tabular}


Table 2. Logistic Regression Predicting Victim Death

\begin{tabular}{|c|c|c|c|c|c|c|c|c|c|}
\hline & \multicolumn{3}{|c|}{ Model 1. Network Time } & \multicolumn{3}{|c|}{$\begin{array}{r}\text { Model 2. Network Dist. } \\
\text { Std. }\end{array}$} & \multicolumn{3}{|c|}{ Model 3. Euclidean Dist. } \\
\hline & $\beta$ & & Std. Error & $\beta$ & & Error & $\beta$ & & Std. Error \\
\hline \multicolumn{10}{|l|}{ Distance Measure } \\
\hline Network Time (min) & 0.022 & $*$ & 0.010 & & & & & & \\
\hline Network Distance $(\mathrm{km})$ & & & & 0.030 & $\neq$ & 0.016 & & & \\
\hline Euclidean Distance $(\mathrm{km})$ & & & & & & & 0.042 & $*$ & 0.021 \\
\hline \multicolumn{10}{|l|}{ Injury Location } \\
\hline Torso & -1.238 & $* * *$ & 0.101 & -1.234 & $* * *$ & 0.101 & -1.236 & $* * *$ & 0.101 \\
\hline Multiple & -1.195 & $* * *$ & 0.097 & -1.194 & $* * *$ & 0.097 & -1.195 & $* * *$ & 0.097 \\
\hline Extremities & -4.876 & $* * *$ & 0.262 & -4.871 & $* * *$ & 0.262 & -4.873 & $* * *$ & 0.262 \\
\hline \multicolumn{10}{|l|}{ Victim \& Situational } \\
\hline Outdoors & -0.551 & $* * *$ & 0.143 & -0.551 & $* * *$ & 0.143 & -0.548 & $* * *$ & 0.143 \\
\hline Clustered Arrivals (15 min) & -0.504 & & 0.318 & -0.519 & & 0.319 & -0.531 & $\neq$ & 0.320 \\
\hline Race White & -0.123 & & 0.103 & -0.13 & & 0.103 & -0.137 & & 0.104 \\
\hline Sex Male & 0.312 & $*$ & 0.158 & 0.308 & $\neq$ & 0.158 & 0.310 & $\neq$ & 0.158 \\
\hline Age & 0.015 & $* * *$ & 0.003 & 0.015 & $* * *$ & 0.003 & 0.015 & $* * *$ & 0.003 \\
\hline Intercept & 0.224 & & 0.196 & 0.227 & & 0.196 & 0.225 & & 0.196 \\
\hline \multicolumn{10}{|l|}{ Model Fit Statistics } \\
\hline AIC & 4071.4 & & & 4073.0 & & & 4072.4 & & \\
\hline $\mathrm{BIC}$ & 4137.6 & & & 4139.3 & & & 4138.7 & & \\
\hline CVE & 0.11979 & & & 0.119849 & & & 0.11983 & & \\
\hline
\end{tabular}

$\neq \mathrm{p}<.1 ; * \mathrm{p}<.05 ; * * \mathrm{p}<.01 ; * * * \mathrm{p}<.001$ 